\title{
Topological Tori as Abstract Art
}

\author{
Carlo H. Séquin \\ CS Division, University of California, Berkeley \\ E-mail: sequin@cs.berkeley.edu
}

\section{Abstract}

Topologists have known for almost three decades that there are exactly four regular homotopy classes for marked tori immersed in 3D Euclidean space. Instances in different classes cannot be smoothly transformed into one another without introducing tears, creases, or regions of infinite curvature into the torus surface. In this paper, a quartet of simple, easy-to-remember representatives for these four classes are depicted to expose this classification to a broader audience. It is shown explicitly how the four models differ from one another, and examples are given how a more complex immersion of a torus can be transformed into its corresponding representative. These mathematical visualization models are juxtaposed with artistic sculptures employing more classical torus shapes.

Keywords: toroidal sculptures, regular homotopies, torus eversion.

\section{Introduction}

Many artists, as long as 5000 years ago, have celebrated tori as art objects. The Naturhistorische Museum in Vienna displays a toroidal bronze object that is about 5000 years old (Figure 1(a)). More recent artists have depicted tori in stone or metal, singly or in intertwined pairs, with spiral cuts or parts missing to expose the cross section, with rough surfaces or with shiny mirror-like finishes (Figure 1).

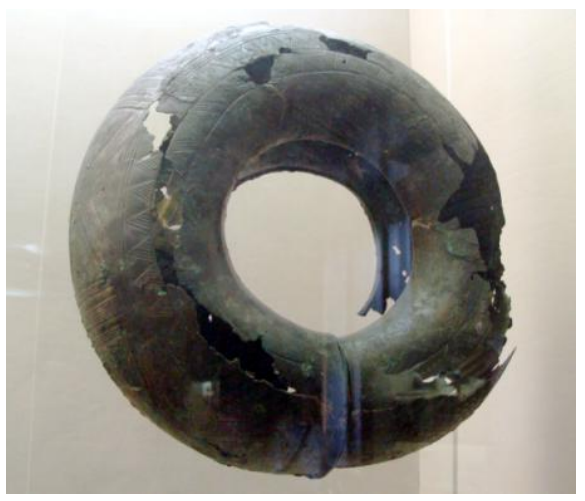

(a)

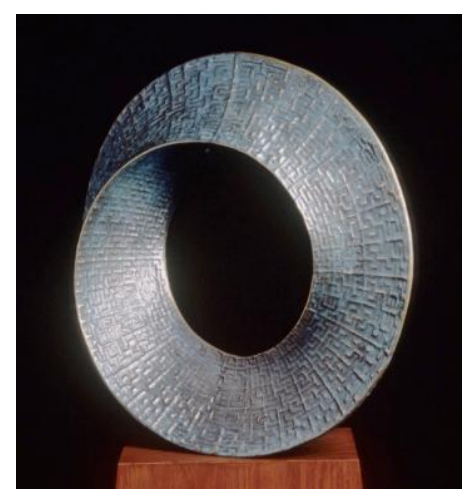

(d)



(b)

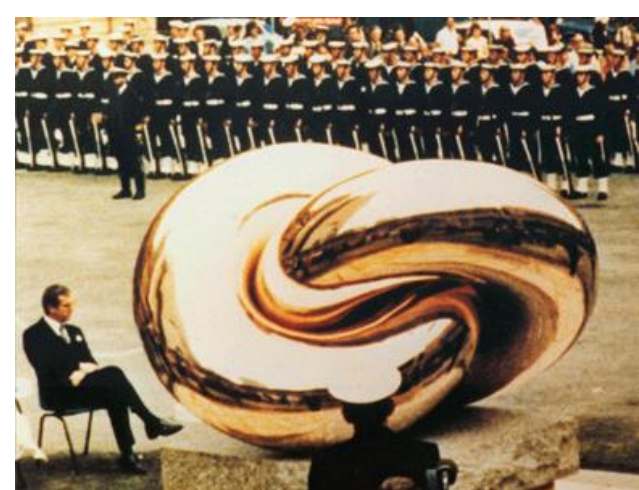

(e)



(c)

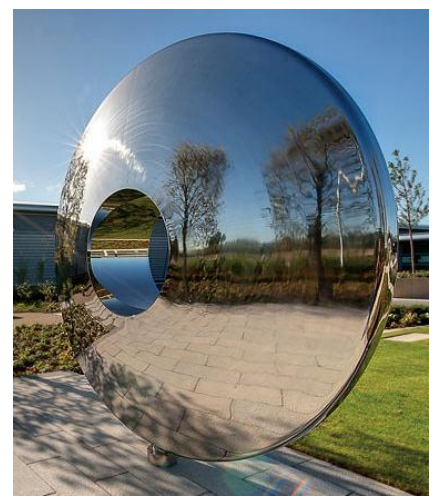

(f)

Figure 1. Tori as art objects: (a) by unknown 5000 year old artists (photo by C.H. Séquin) and by (b) Keizo Ushio (from [25] used by permission), (c) Jonathan Prince (from [16] used by permission), (d) Helaman Ferguson (from [6] used by permission), (e) John Robinson (from [18] used by permission),

(f) David Harber (from [8] used by permission). 
However, to a mathematician tori may assume much more involved geometries than the simple donut shapes underlying the above art works. Topologically, a torus is any surface that results from closing a rectangular domain onto itself by joining opposite edges, while observing their orientation (more on that in Section 2). In this closure process the surface may undergo arbitrary smooth deformations and may even pass through itself; connectivity is more important than geometry. Here we concern ourselves with immersions of tori in 3D Euclidean space $\left(\mathbf{R}^{3}\right)$. In this context, every small neighborhood of the surface must remain homeomorphic to a disk, and no sharp creases or other points of infinite curvature must ever be formed. Figure 2 to 4 give examples of the fancy shapes that can be assumed by such immersions of a torus. The toroidal sweep path may be knotted (Figure 2(a)), it may form an internally knotted tunnel (Figure 2(b)), or it may form multiple loops that partially pass through one another (Figure 2(c)).

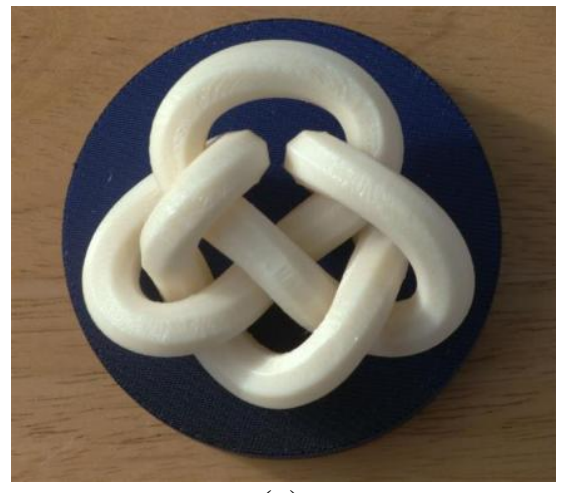

(a)

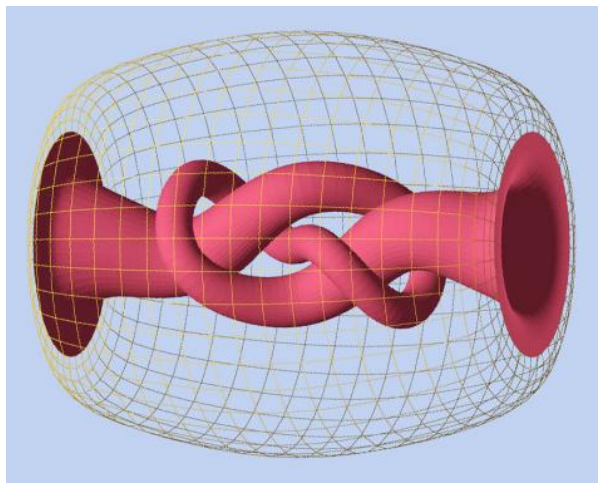

(b)



(c)

Figure 2. Non-trivial toroidal sweep paths: (a) Knot $7_{7}$; (b) tunnel in the shape of a figure- 8 knot; (c) self-intersecting trefoil loop.

Moreover, the cross section used in the toroidal sweep need not be circular. It may form a fancy lobed shape as in Sullivan's 7-fold Hopf torus [24] (Figure 3(a)), or it may be a self-intersecting figure-8 shape (Figure 3(b)), or even form a curtate hypocycloid (Figure 3(c)).

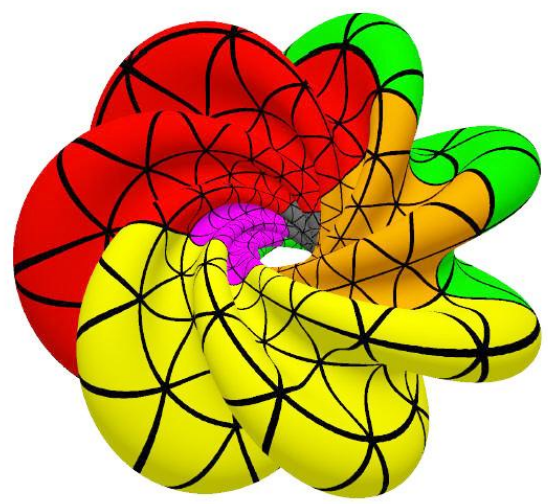

(a)



(b)

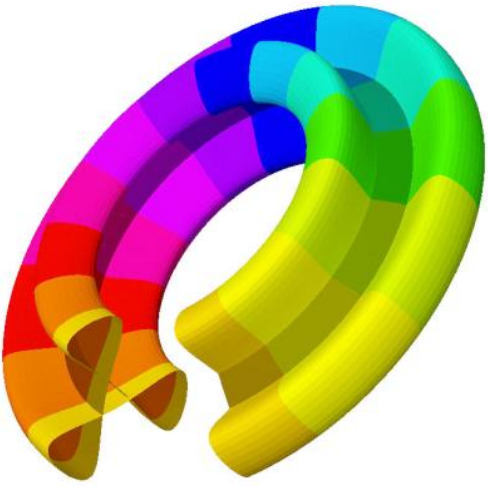

(c)

Figure 3. Non-circular torus cross-sections: (a) a lobed shape (from [24], used by permission), (b) self-intersecting figure-8 shape, (c) curtate hypocycloid profile.

Furthermore, the torus may have external or internal collars (Figure 4(a), (b)), or it may have an even number of local surface inversions as they appear in the mouth of the classical Klein bottle (Figure 4(c)). All of these convoluted shapes are proper immersions of marked (decorated) tori. 


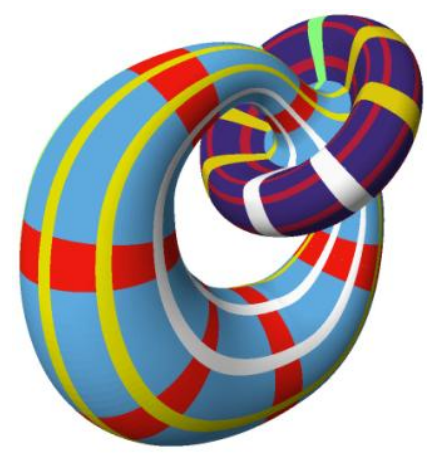

(a)

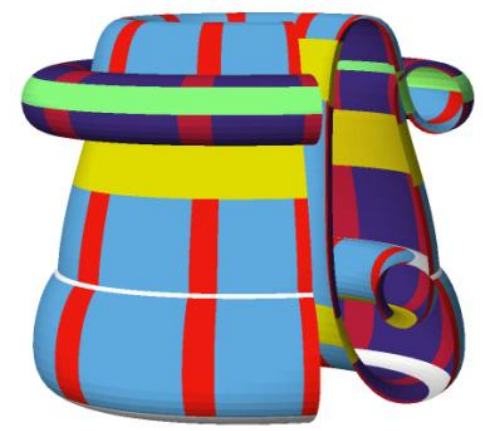

(b)

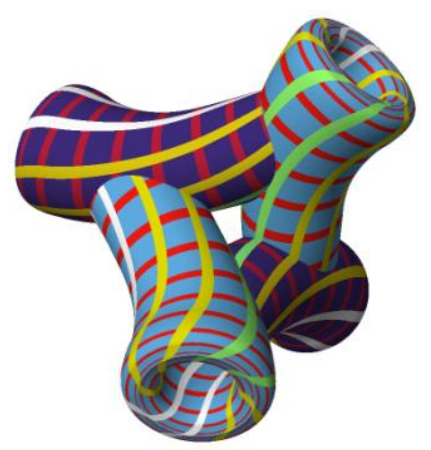

(c)

Figure 4. Weird Tori: (a) with a single outside collar, (b) with inside and outside collars, (c) with four Klein-bottle mouths turning the surface locally inside out.

The question now arises, if we were to use the same texture map on the underlying rectangular domain, which of these decorated tori could be smoothly deformed into one another, and which ones would belong to different regular homotopy classes.

\section{Regular Homotopies of Tori}

Shapes that can be smoothly transformed into one another are said to be related by a regular homotopy; they belong to the same regular homotopy class. In all transformations within one and the same class, surface regions are allowed to pass through one another, but surfaces may not be punctured, cut, or torn, and they must neither assume any sharp creases nor form any other singular points with infinitely sharp curvature. A paper by Hass and Hughes [9] shows that orientable 2-manifolds of genus $g$, immersed in Euclidean 3D space $\left(\mathbf{R}^{3}\right)$ and marked with some kind of parameter grid, fall into $4^{g}$ regular homotopy classes. For genus zero there is only a single class. Thus all "blobs" are the same, and a sphere can be turned inside-out through a sequence of regular homotopy moves. This was first proven by Steve Smale in 1958 [22], but it took many years before a first sequence of actual moves was published [15]. A more elegant sphere-eversion process was later conceived by Bernard Morin [14], and it led to a first computergraphics movie of that transformation created by Nelson Max [13]. The same basic series of moves was later optimized in the film Optiverse [23] so as to minimize the maximal amount of surface bending energy reached during this process. Another sphere eversion process relying on Dirac's Belt Trick has become well-known through the video Outside-In [10].

Based on that same paper [9], one expects four different regular homotopy classes for the torus, where the representatives in one class cannot be turned into that of another class through any regular homotopy-preserving moves. While a lot of work has been done to depict the above mentioned sphere eversions, much less work has been done for the torus to visualize its four regular homotopy classes and possible transformations within them. This paper presents depictions of four simple representatives of those four classes in a way that should be accessible to readers without expert knowledge in topology or group theory. It also introduces examples of regular homotopies that allow one to take a more complicated torus and transform it into one of those four forms.

Of course, it is somewhat a matter of taste as to which particular shape is the most "natural" representative of a particular regular homotopy class, since any shape within that class might be selected. I was looking for shapes that can easily be visualized and remembered and in which the parameterization closely follows the natural lines of curvature of the chosen shape - without any additional twisting. Following suggestions by John Sullivan and Craig Kaplan, I have introduced a corresponding, intuitively plausible labeling at the Bridges conference [19]. For a better understanding of this choice, I will first review a simple, way to form an immersed torus, which is general enough so that representatives of all four regular homotopy classes can be constructed. 


\section{Constructing a Parameterized Torus}

An immersed torus can be formed from a rectangular domain, in which opposite edges are joined while observing their directionality. The surface may pass through itself, but it must remain smooth, and any local neighbourhood on this surface must maintain the connectivity of a small disk. In a first step we smoothly join the vertical edge pair, annotated with green arrows in Figure 5(a), forming some kind of a generalized cylinder. In doing this we have quite a few options: We may roll the rectangle backwards (Figure 5(b)) - or forward, thereby exposing its back side. (The back of this rectangle carries the same (mirrored) pattern but with a more purplish hue and darker shading.) Alternatively, we may also let the rectangle self-intersect and form a figure-8 shaped cross section (Figure 5(c)), or we can roll the sheet into a double cylinder (Figure 5(d)) or into some other multi-walled, self-intersecting tube.

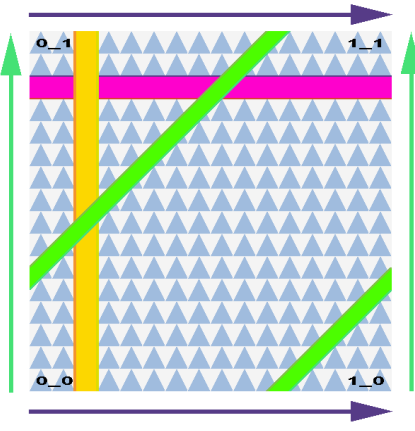

(a)

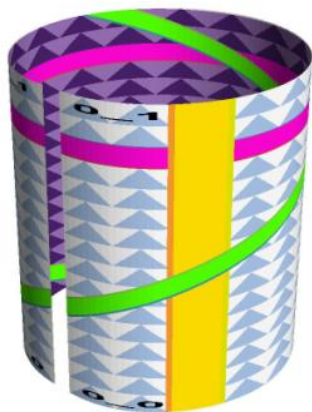

(b)



(c)

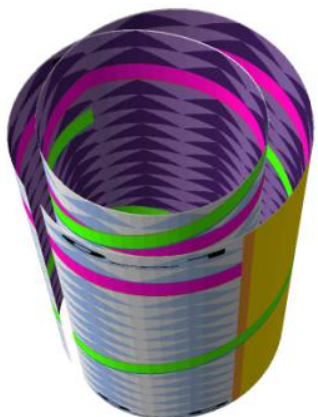

(d)

Figure 5. Step \#1 of forming a torus by folding up a rectangular domain (a): Merging the two vertical edges: (b) into a cylinder, (c) into a self-intersecting figure-8 shape, or (d) into a multiply-rolled tube.

In a second step we then smoothly join the ends of this generalized tube into some closed loop; this corresponds to joining the horizontal, purple arrows in Figure 5(a). Again, we have quite a bit of freedom how to accomplish this: We can form a simple donut (Figure 6(a)), or we can add $360^{\circ}$ of twist before we close the ring (Figure 6(b). The sweep path of the emerging loop may be circular, or it may form multiple loops, a self-intersecting figure-8 shape (Figure 6(c)), or more complex knots or tangles (Figure 6(d)).

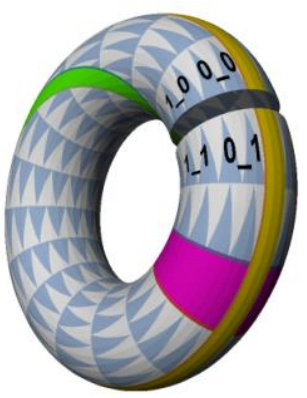

(a)

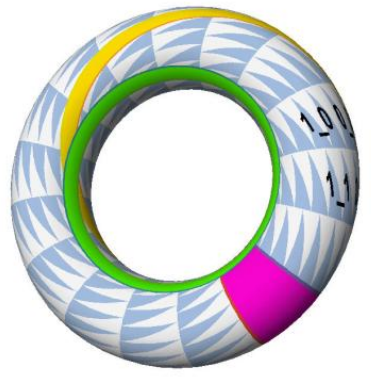

(b)

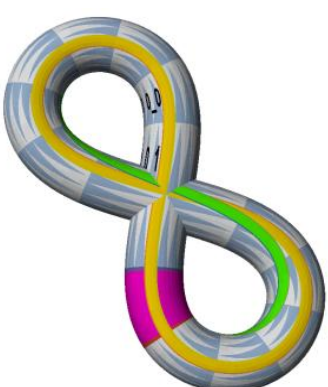

(c)

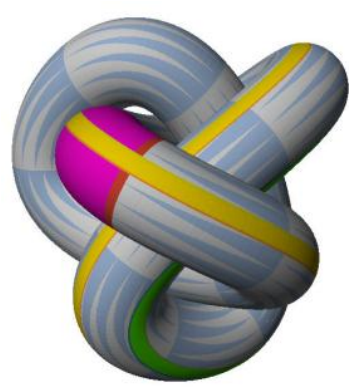

(d)

Figure 6. Step \#2 of folding up a rectangular domain into a torus: Merging the horizontal edges to close the tube: (a) on a circular path with no twist, (b) with $360^{\circ}$ twist, (c) along a figure- 8 path, and (d) along an arbitrary tangled space curve.

In the first step we might even elect to give the rectangular domain a longitudinal twist of even multiples of $180^{\circ}$ before we join its two vertical, green edges (Figure 7(a)), and we would still be able to close the surface into a legal torus (Figure 7(b)). However, this is not the primary choice, since our goal is to construct simple representatives with the least twisted parameterization. Later in the paper it will be shown how we can deal with more heavily twisted tori. 


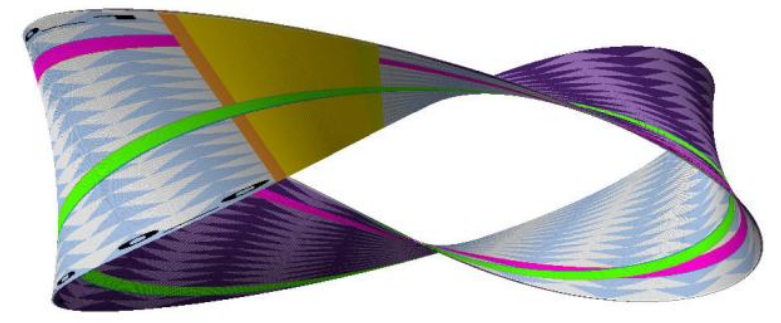

(a)

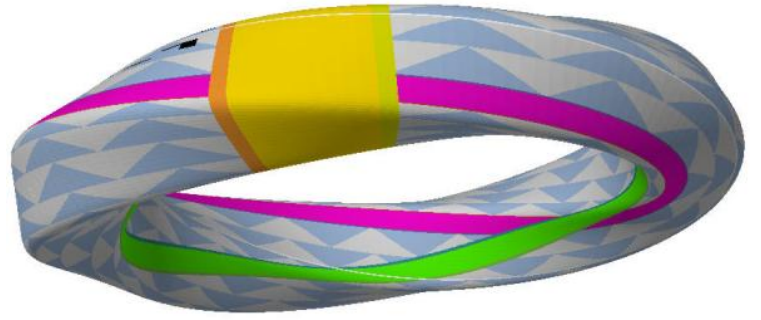

(b)

Figure 7. (a) Adding a twist into step \#1; (b) closing the surface into a twisted torus.

In all of the above steps we are not just concerned with creating smooth, closed shapes without any borders, but we also keep track of what happens to the decoration on the original rectangular domain (Figure 5(a)). If we assume that the original domain is marked with a rectilinear parameter grid, then it is required that all those parameter lines, e.g., the red and yellow bands in Figures 5 and 6 , close onto themselves. On an ordinary torus (Figure 6(a)) the lines of curvature provide a natural parameterization grid. However, if the sweep path is not planar, but an arbitrary 3D space curve as in Figure 6(d), a torsionminimizing parameterization will generally produce "longitudinal" lines (running perpendicularly to the profile curves) that do not close smoothly around the toroidal loop, and which would require some amount of twist (around the sweep path) to keep these lines connected to themselves. In order to clearly define a nomenclature for describing the surface parameterization, we will use a torus with a planar sweep path as a reference. The plane containing that sweep path is called the equatorial plane. The profile curves that are being closed during the first step (Figure 5), and which are now lying in planes perpendicular to the tube axis, are called meridians. If the cross section remains constant and is swept in a torsion-minimizing manner, then the second set of parameter lines will lie in planes that are parallel to the equatorial plane, and thus they naturally can be called parallels. Topologists call these lines "longitudes", which may be a bit confusing, considering that on the globe the lines of longitude are called meridians! We also call the few special parallels that lie in the equatorial plane equatorials. Moreover, there also exist diagonal lines that close onto themselves. They combine integer numbers of loops along a meridian and along a parallel. On an ordinary torus, a (1,1)-diagonal, e.g., the green ribbon in Figure 6(a), will travel exactly once around the major and minor circles, respectively.

\section{Four Generic, Un-twisted Representatives}

An easy way to obtain four different representatives for the four regular homotopy classes, makes direct use of the 2-step torus construction described above. It turns out that we can obtain four different generic representatives of the four regular homotopy classes of all possible tori by distinguishing the following four cases: We either use a circular cross section $(\mathbf{O})$ or one in the shape of a figure-8, and we close the tube either along a circular sweep path $(\mathbf{O})$ or along a figure- $\mathbf{8}$ curve [19][20].

In all four cases the major curve along which a circular or a figure- 8 cross section has been swept to form the torus lies in a plane (Figure 8). Thus, when a torsion-minimizing surface parameterization is applied, the parameterization grid (indicated by the displayed texture) shows no unnecessary twisting and follows the natural lines of curvature; all parallels nicely close onto themselves, and so do the three characteristic ribbons shown: the ribbon along a meridian (magenta, red), the ribbon along a parallel (yellow), and an additional $(1,1)$-diagonal ribbon (green). If we could peel off these characteristic ribbons, and spread them out into circular loops, we would find that some of them are twisted, even though the underlying torus shows no twist. In particular, the yellow parallels on tori of types TO8 and T88 show $360^{\circ}$ of twist, as do the red meridians on types $\mathbf{T 8 O}$ and $\mathbf{~ T 8 8}$, as well as the green diagonals on types TOO and T88. 


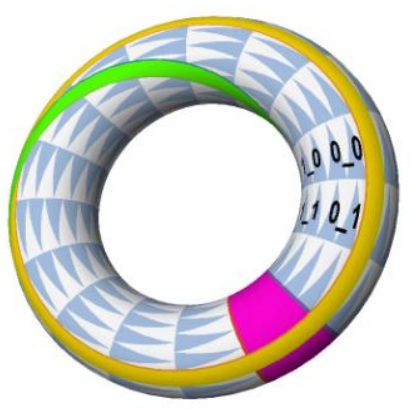

TOO



TO8



T80



T88

Figure 8. Tori in four different homotopy classes, characterized by their profile and sweep path $(\mathbf{O} / \mathbf{8})$, with 3 embedded characteristic ribbons: meridians (red), parallels (yellow), (1,1)-diagonals (green): Type TOO: meridians: untwisted, parallels: untwisted, (1,1)-diagonals: $360^{\circ}$ twist; Type TO8: meridians: untwisted, parallels: $360^{\circ}$ twist, $(1,1)$-diagonals: untwisted; Type T80: meridians: $360^{\circ}$ twist, parallels: untwisted, (1,1)-diagonals: untwisted; Type T88: meridians: $360^{\circ}$ twist, parallels: $360^{\circ}$ twist, (1,1)-diagonals: $360^{\circ}$ twist.

Readers may wonder why the shapes TOO and TO8 are representing different homotopy classes, since it should be easy to un-warp the figure-8 loop into a plain circular path, given that topology allows surface parts to pass through one another. Here is where the surface decoration plays an important role. As we change the shape of the sweep path in 3D space, the skin of the torus may experience some twisting. In particular, when we change the path from a figure- 8 shape to a circle, the torus surface is twisted by $\pm 360^{\circ}$ around the sweep path (Figure 9(c)) - with the sign depending on the direction in which we move the two crossing branches apart. The result is equivalent to a regular torus on which a Dehn twist [5] of $360^{\circ}$ along a meridional cut line has been introduced; we thus call this an M-twist. In this twisting operation all meridional ribbons (magenta) will remain untwisted.

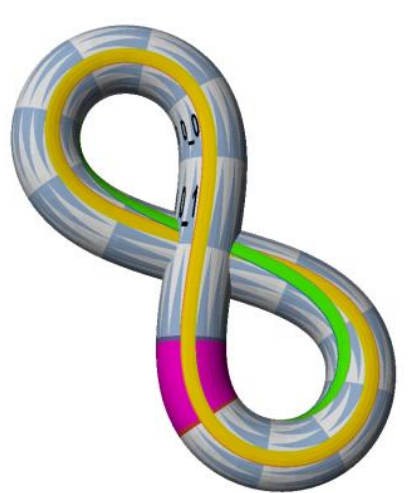

(a)

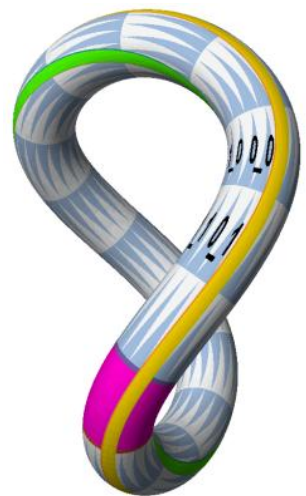

(b)

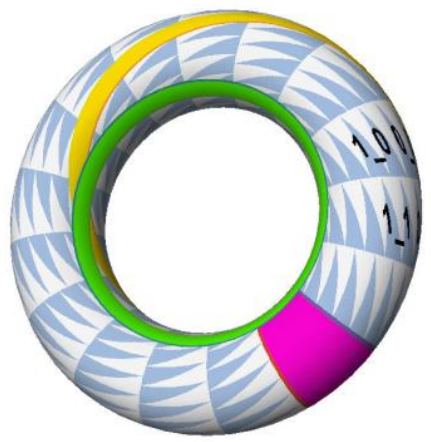

(c)



(d)

Figure 9. (a) $\rightarrow$ (c) Untangling a torus of type TO8 starting with a downward move of the torus branch with the yellow-green ribbon crossing; (d) unwrapped torus surface texture.

When we examine the surface of the torus shown in Figure 9(c), the green diagonal ribbon has turned into a parallel ribbon, and the yellow ribbon has become a (-1,1)-diagonal (Figure 9(d)). If we had separated the crossing torus branches in the opposite directions, then the yellow ribbon would have become a $(1,1)$ diagonal, while the green ribbon would have turned into a $(2,1)$-diagonal; this would show up on the torus surface as a $(1,2)$-torus knot with a total twist of the corresponding ribbon of $720^{\circ}$. The readers are encouraged to take a long, thin, physical paper strip, to model it in the shape of any of the above colored surface ribbons, and then to verify the amount of twisting found in the ribbon. 


\section{Figure-8 Sweep Cross-Over Move}

By going through the regular homotopy transformation starting from Figure 9(c), passing through Figures 9(a) and 9(b), and by continuing this process until we have again a perfectly round torus, the (meridional) twist in the toroidal loop has been changed by $720^{\circ}$, but the torus remains in the same regular homotopy class. We call this whole sequence of transformations a Figure-8 Sweep Cross-over Move. By repeating or reversing this move, we can always add or subtract meridional twist in increments of $720^{\circ}$. Thus for our classification of torus immersions, we will count twists modulo $720^{\circ}$. Therefore, any built-in toroidal twist is either zero or $360^{\circ}$, and these are the only two relevant values.

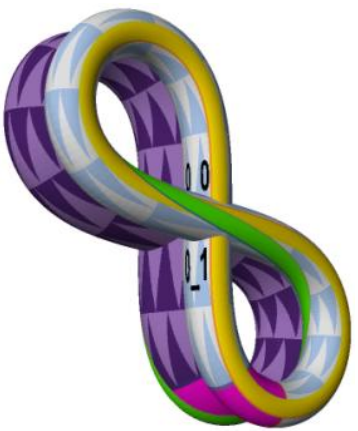

(a)

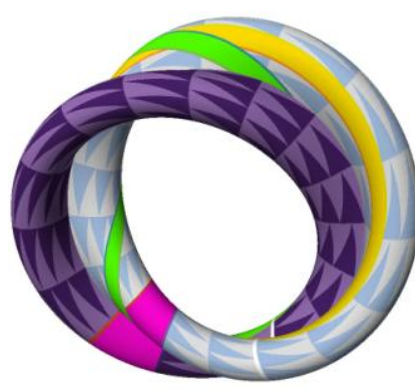

(b)

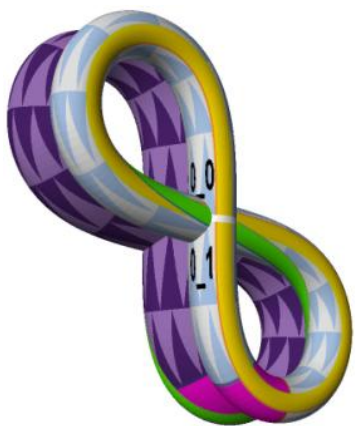

(c)

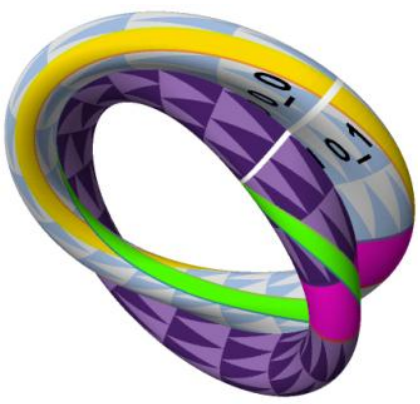

(d)

Figure 10. Untangling the figure-8 path of type T88. (a) $\rightarrow$ (b) result of moving the green/yellow crossing upwards; (c) $\rightarrow$ (d) result of moving the green/yellow ribbon-crossing downwards.

The Figure-8 Sweep Cross-over Move can also be used to untangle the central crossing in tori of type $\mathbf{T 8 8}$ (Figure 10(a)). Again, depending on the way in which the crossing strands are moved apart in order to separate them, a twist of $\pm 360^{\circ}$ will have been introduced by the time the figure- 8 path has been unwound into a planar, circular loop. For this surface the difference between a negative twist (Figure 10(b)) and a positive twist (Figure 10(d)) is much more obvious, since it shows up in the surface geometry itself, and not just in its parameterization. The regular homotopy moves leading from Figure 10(b) through Figures 10(a) and 10(c) to Figure 10(d) will again result in a total twist change of $720^{\circ}$.

\section{Analysis of the Twistedness of Characteristic Ribbons}

Because of the transformations described above, the twistedness built into a closed ribbon is either zero (an even multiple of $360^{\circ}$ twist) or one (an odd multiple of $360^{\circ}$ twist). Regular homotopy transformations cannot change the amount of built-in twistedness. Thus tori that have different twistedness in corresponding ribbons must belong to different regular homotopy classes. For our classification it is sufficient to analyze two characteristic ribbons: one along a meridional parameter line, and one along a longitudinal line. Either one of these ribbons may either be twisted or untwisted, and there are exactly four possible combinations. Four corresponding representatives are depicted in Figure 8, and the twistedness of their characteristic ribbons is explicitly stated in the caption. Analyzing the amount of twisting found in a complementary set of characteristic ribbons will always allow us to classify any arbitrarily convoluted marked torus.

\section{Other Interesting Regular Homotopies}

\section{Turning a Torus Inside-Out}

On the web one can find several references to the process of Torus Eversion - not all of which describe regular homotopy transformations; in particular, the classical eversion through a puncture in the surface [12] is not admissible for our purposes. However, Arnaud Chéritat presents an elegant and easy-tounderstand video that shows a proper regular homotopic eversion [2]. A closely related process is described in diagrammatic form by [1]. Figure 11 shows my own diagrammatic depiction of this process. 


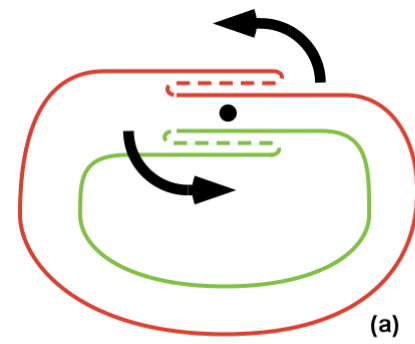

(a)

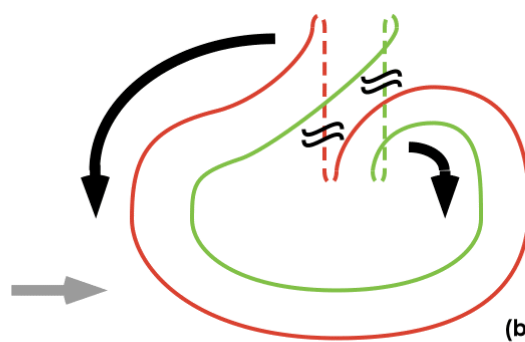

(b)
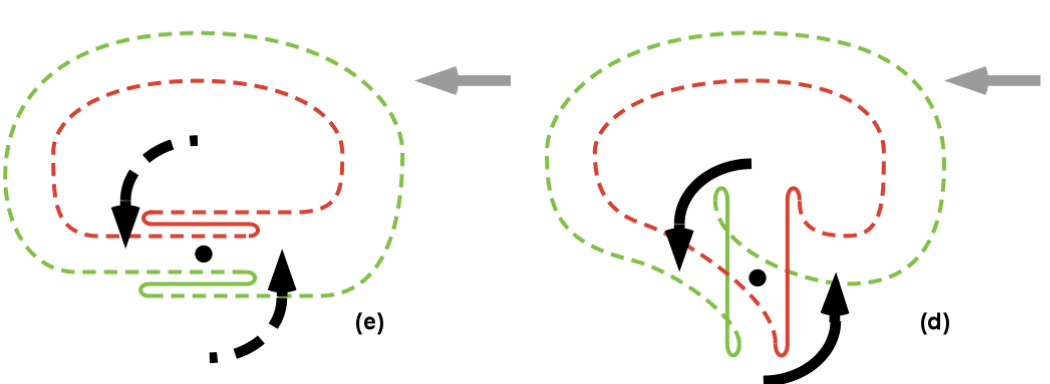

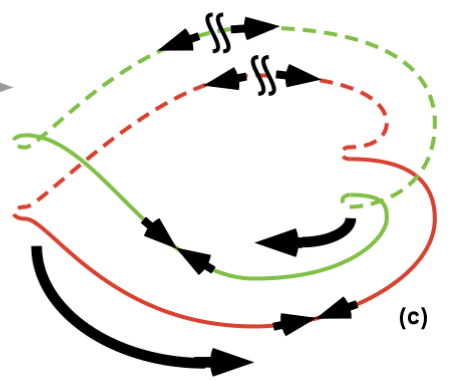

(c)

Figure 11. Turning a torus inside out: (a) $\rightarrow$ (e) schematic view of two parallels (equatorials).

During the whole process, the sweep curve remains planar, and the whole transformation can thus be depicted by simply drawing two opposite parallels (two equatorials), shown in (dark) red and (light) green, respectively, which are the intersection lines of the torus with the equatorial plane. Portions of the torus, where surface regions that were originally facing inward become exposed towards the outside, are shown as dashed lines; this makes it obvious that the torus gets turned inside-out.

The process starts by introducing a rotationally symmetrical 3-layer fold around some meridian in the torus (Figure 11(a)). The resulting everted tube segment is then turned through $90^{\circ}$ (Figure 11(b)) and further turned and stretched, so that the two "Klein-bottle mouths" that delimit this segment can be moved apart (Figure 11(c)). They are moved in opposite directions around the toroidal loop. On the other side of the loop they are recombined into a short, straight tube segment (Figure 11(d)). This tube segment is then turned into alignment with the toroidal ring (Figure 11(e)) and unfolded in place.

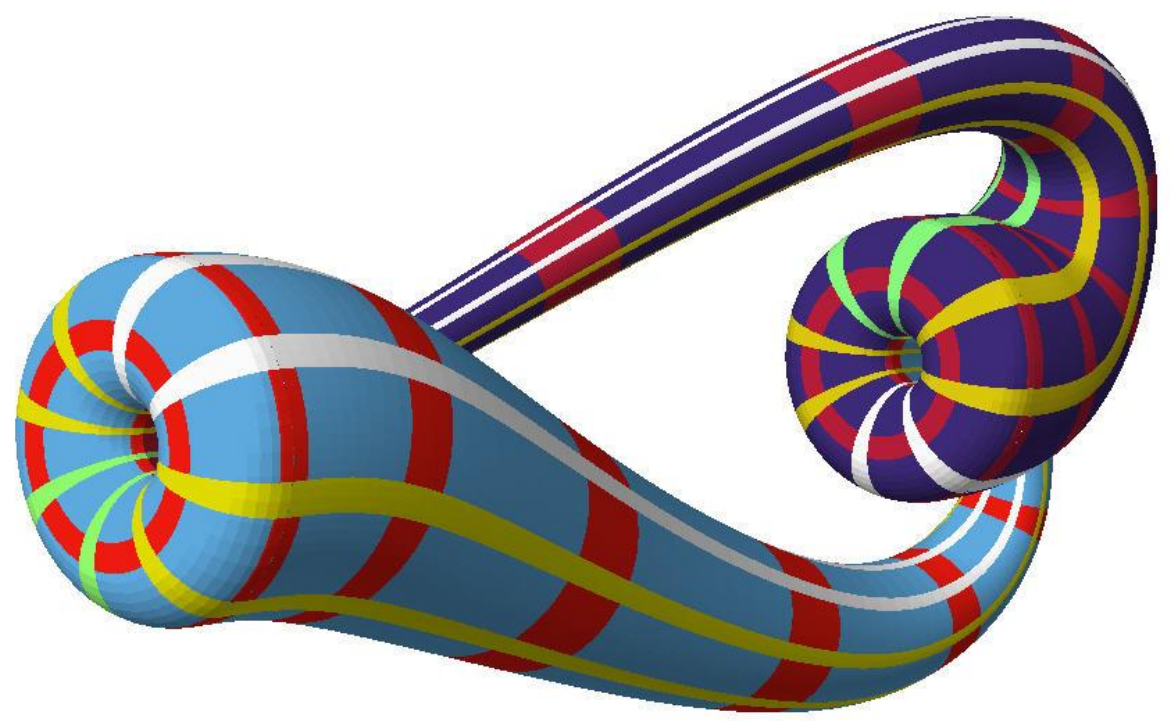

Figure 12. Torus eversion halfway-point corresponding to Figure 11(c). 
All these moves are "planar" operations, and thus they introduce no twisting of any kind. The triple fold introduced in Figure 11(a) can also readily be generated for a tube with a figure- 8 cross-section. Moreover, the two Klein-bottle mouths have no problem passing through the (topologically irrelevant) cross-over intersection generated by a figure- 8 sweep path. Thus this eversion process is directly applicable to tori of all four regular homotopy types. Figure 12 shows the half-way point (Figure 11(c)) in a more "physical" 3D manner. From an artistic point of view this also forms a pleasing heart shape.

\section{Changing Parameterization and Profile}

Another planar transformation that introduces no twist allows us to swap the parameterization, i.e., to exchange the roles of parallels and meridians (Figure 13). We introduce a triple fold into a torus of type TO8 and maintain the resulting inverted tube segment as the core of a future "tubular" torus. By sliding the lobes of the figure- 8 loop next to the inverted segment trough one another (Figure 13(b)), we obtain a simple loop connected to the inverted segment (Figure 13(c)). When we let this loop contract, we realize that the new meridians formed have a figure- 8 shape and thus are twisted (Figure 13(d)). The result thus is a type T80 torus with swapped parameterization. In Figures 13(e) the red bands were meridians; in Figures 13(f)) they now represent parallel parameter lines.

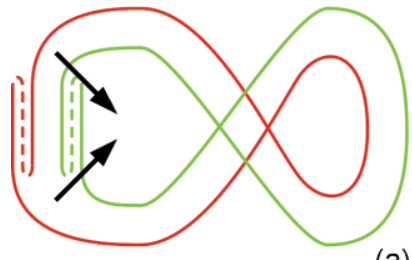

(a)



(e)

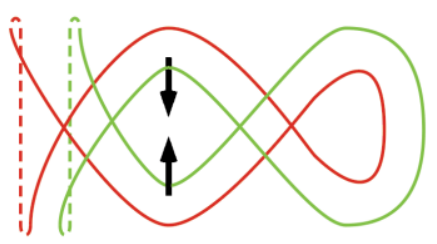

(b)

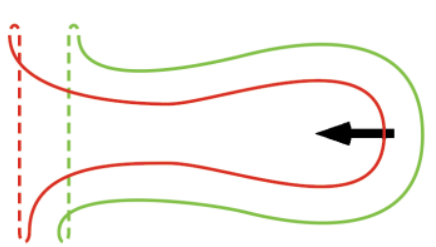

(c)



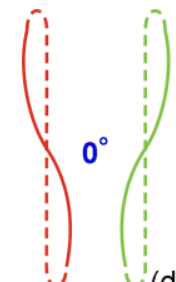

(d)

(f)

Figure 13. Swapping the parameters is equivalent to switching between torus Type TO8 and Type T8O; (a) $\rightarrow$ (d) schematic transformation; (e, f) 3D models of the start and ending of the transformation.



(a)

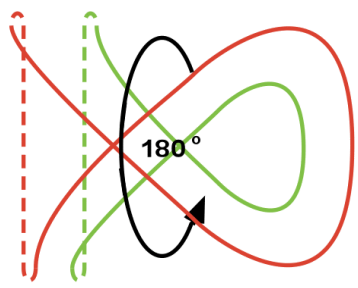

(b)


(c)

Figure 14. Turning a torus of type TOO into type T8O with a twist and swapped parameterization.

If we try to do a similar operation on a torus of type TOO (Figure 14(a)), we find that we cannot collapse the loop without moving its sweep path out of the equatorial plane. The loop has to be un-tangled by going through the $3^{\text {rd }}$ dimension (Figure $14(\mathrm{~b})$ ). This $180^{\circ}$ flip introduces an M-twist of $360^{\circ}$ into the 
contracting tube segment (Figure 14(c)), which then manifests itself as a Dehn twist of $360^{\circ}$ along a longitudinal cut line, which we call L-twist. The previously untwisted parallels have now become untwisted diagonals in this "T8O torus with $360^{\circ}$ L-twist". Overall we have achieved a swap of the parameterization combined with a profile change and the introduction of $360^{\circ} \mathrm{L}$-twist.

\section{Adding Dehn Twist}

At this point it is worthwhile to point out that there is a simple operation to add any incremental L-twists of $\pm 720^{\circ}$, which would be equivalent to a "cut-twist-reconnect" operation along a longitudinal cut line. Figure 15 shows how one can grab the inner wall of a tubular torus, pull it sideways through the outer wall, and subject it to a complete Figure-8 Sweep Cross-over Move (Figures 15(b) and 15(c)). This will introduce $\pm 720^{\circ} \mathrm{M}$-twist into this tubular loop, which then results in $\pm 720^{\circ}$ L-twist in the final torus.
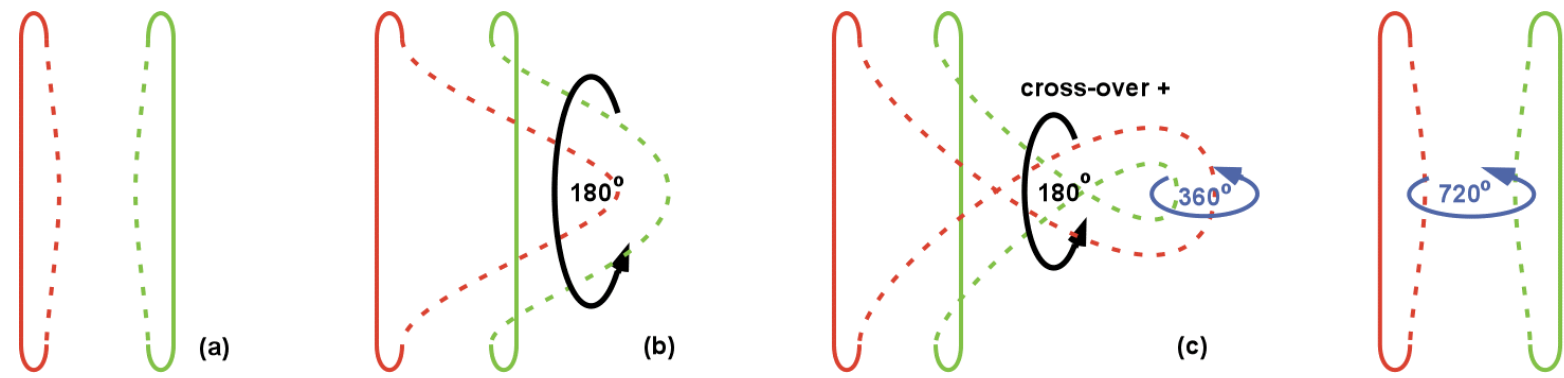

(d)

Figure 15. Introducing an incremental L-twist of $\pm 720^{\circ}$ with two half-flips of a loop of the "inner tube."

\section{Map of All Four Regular Homotopy Classes}

After the discussion of a few examples of regular homotopy moves that do not change the classification of a particular torus, we now present the complete map of the four regular homotopy classes and of the discrete changes to the parameterization that do effect a change in classification (Figure 16). For instance, we can cut a torus along a meridian or along a longitudinal parameter line and introduce $360^{\circ}$ of Dehn twist along that cut. On a plain torus (TOO) this will clearly change the twistedness of any ribbon that crosses this cut line. For any M-twist introduced in this manner, we can use a Figure-8 Sweep Cross-over Move (Figure 9) to undo the twist in the yellow ribbon; but in doing so, we change the "TOO with twist" into a pure TO8. Similarly, we can undo the effects of an L-twist, visible in the red ribbon, with a move as shown in Figure 14; but in doing so, we change the torus into type T8O.

On the other hand, if we cut a $\mathbf{T 8 O}$ torus along a figure- 8 meridian, the introduction of $360^{\circ}$ of Dehn twist will cause one end of a crossing longitudinal (yellow) ribbon to travel around this curve of turning number 0 , and this will not change its twist. That is why some twist operations on certain tori leave the type of the torus unchanged; such operations are indicated in Figure 16 by double arrows that loop back to the same torus type. This means that such a parameter change can be achieved through a regular homotopy move.

Figure 16 shows how the world of tori splits into the four different classes. It shows the four types of tori symbolically in the four gray circles, including a schematic diagram of the geometry of the key representative and a characterization of three characteristic ribbons. The meridians and parallels in TOO are untwisted (labelled by " $u$ "). But if we connect a paper strip into a figure-8 loop without any twisting and then open it into a circular loop, we find that the ribbon now shows a $360^{\circ}$ twist (labeled by " $t$ "). Since we can always add or subtract twist in increments of $720^{\circ}$ with a Figure-8 Sweep Cross-over Move, "t" and " $u$ " are the only two states a ribbon can assume. Since the four representatives shown have different combinations of twistedness for their characteristic ribbons, and since regular homotopies cannot change the twistedness of a ribbon, the four representatives must indeed belong to different classes. 


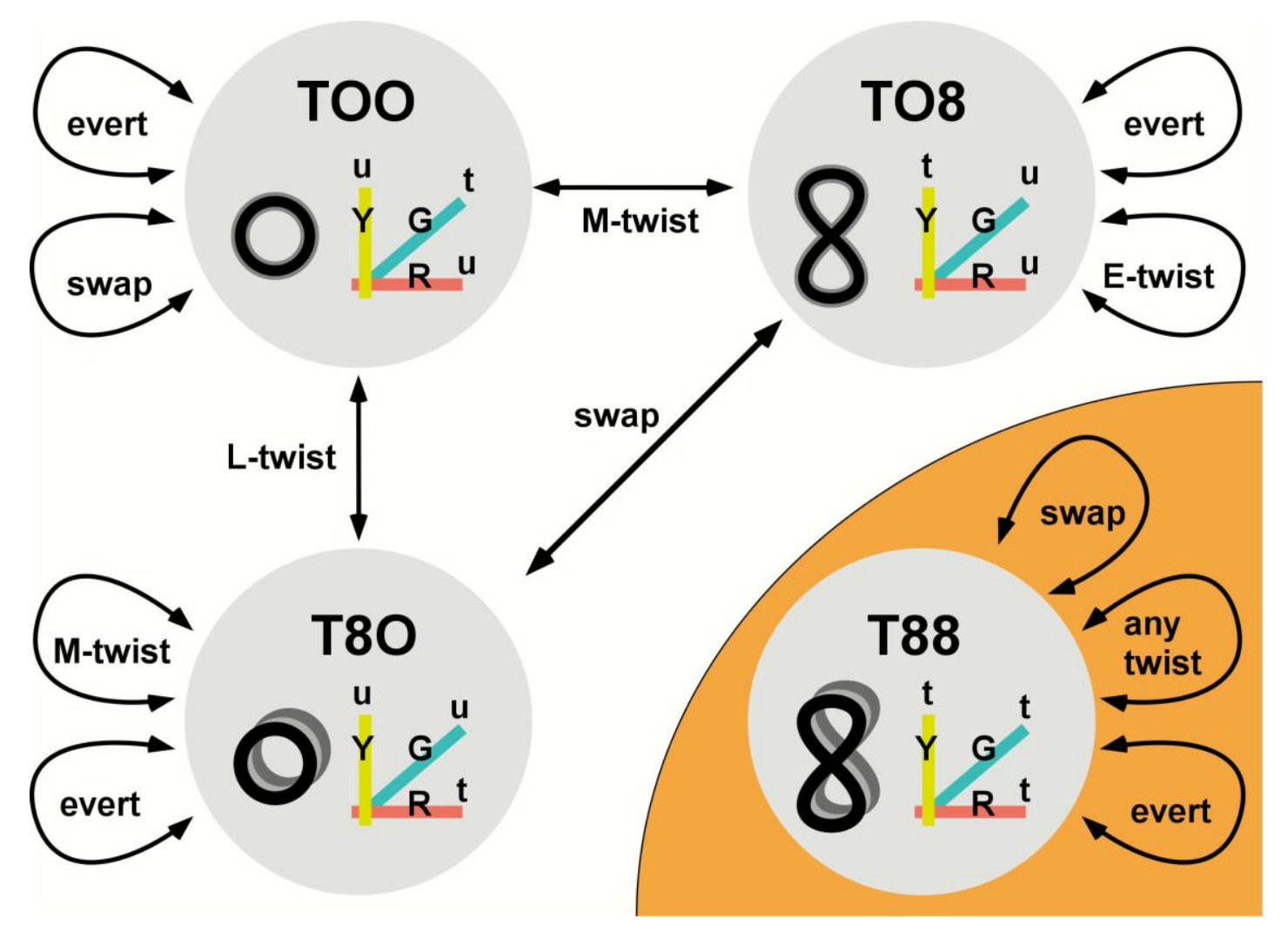

Figure 16. Complete map of the effects of re-parameterizations on the four regular homotopy classes of marked tori in 3D Euclidean space.

The double-headed arrows in Figure 16 indicate the many changes that can possibly be applied to the parameterization of the torus. In addition to adding Dehn twist, which corresponds to a shear operation on the surface "texture" of the torus, we could also rotate the parameterization by $90^{\circ}$ or exchange the role of the meridional and parallel directions, which both amount to "swap" operations in Figure 16. Finally, we could mirror one of the two coordinate axes, which would reverse the direction of their cross product; thus this is equivalent to turning a torus inside out, which is marked as "evert" in Figure 16. The evert operation leaves all tori in their own respective regular homotopy class, as was discussed in Section 3.

It is worth noting that this world of tori partitions into two separate domains (TOO+TO8+T8O versus T88) and that there are no double arrows between these two domains. This means that if we ignore parameterization, there are only two regular homotopy classes for unmarked tori.

\section{Analysis and Simplification of Arbitrary Torus Structures}

The principle for constructing a torus presented with Figures 5 and 6 permits the construction of rather wild and amazing geometries. Some of them are presented here for the enjoyment of the reader. Given an arbitrary, highly contorted torus also presents the challenge of finding out what regular homotopy class it belongs to! Many of the simpler cases can be analyzed by applying some of the fundamental transformations introduced above. But there are perfectly legal tori structures whose shapes go way beyond some simple twisting and/or re-parameterization, and they will require some additional tricks to be converted into one of the four generic representatives. This section presents a series of case studies of increasing complexity to give the reader a richer set of tools for the analysis and understanding of toroidal structures. 


\section{Multi-Loop Tori}

A tube of any given cross-section can be coiled through multiple loops before its ends are joined to form a topological torus. An intriguing example is shown in Figure 17(a). Here a tube with a roughly triangular cross section is coiled into a self-intersecting double loop and closed with an overall twist of $360^{\circ}$. To better understand this structure and its cross section, it is also shown in a segmented version (Figure 17(b)).

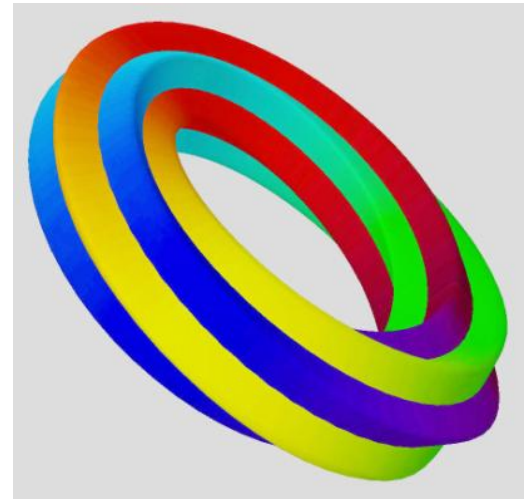

(a)

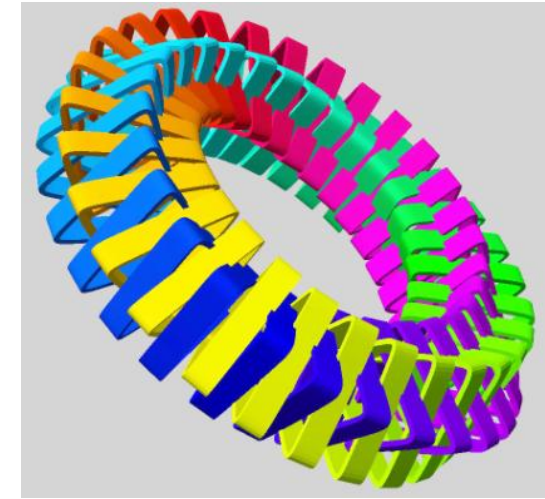

(b)



(c)

Figure 17. (a) Doubly-looped torus with triangular cross section and $360^{\circ}$ twist; (b) the same torus segmented for easy visualization; (c) the same structure after unwinding it into a circular sweep.

Such a doubly-looped structure can be unfolded smoothly into a figure-8 shape (Figure 18). The azimuth of the texture in the moving branch-loop gradually changes through $180^{\circ}$, and the black-white longitudinal ribbon on the moving loop shifts gradually from the inside of the loop to its outside in Figures 18(b) and 18(c). However, there is no net twist introduced into the tube anywhere. On the other hand, if we now untwist the figure-8 path into a simple circle, with half of a Figure- 8 Sweep Cross-over Move (Figure 9), the tube will experience an incremental twist of $360^{\circ}$. In the torus shown in Figure 17, this incremental twist-change cancels out the original twist, so that the final result is just a twist-free torus of type TOO (Figure 17(c)).



(a)

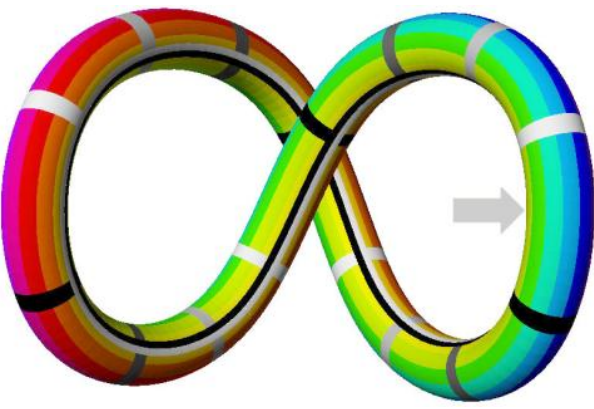

(b)

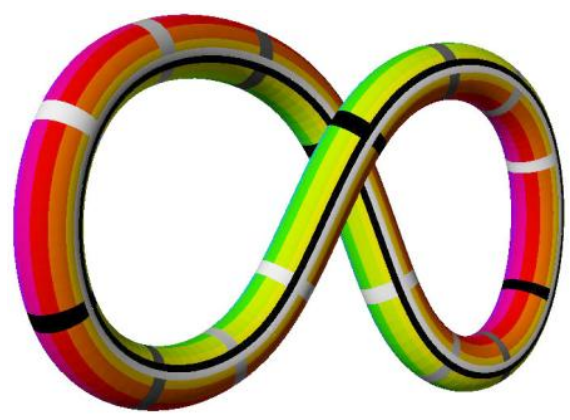

(c)

Figure 18. (a) Unwinding a double loop into a figure-8 path without changing the twist of any characteristic ribbon: (a) starting state, (b) halfway point, (c) final state.

\section{Multi-Rolled Tubes}

Figure 19(a) shows another intriguing torus, with a profile resembling a curtate hypocycloid. In Figure 19(b) the loop has been cut open and its twist removed, so that one can see its cross section. Figures 20(a) to 20 (c) show that this cross section profile, exhibiting a turning number of 2 , can be smoothly 
transformed into a double circle. So the basic structure of this tube is just a doubly-rolled cylinder (Figure 20(d)), which we have encountered already in Figure 5(d). Now we want to turn this torus shown in Figure 19(a) into one of the four generic representatives.

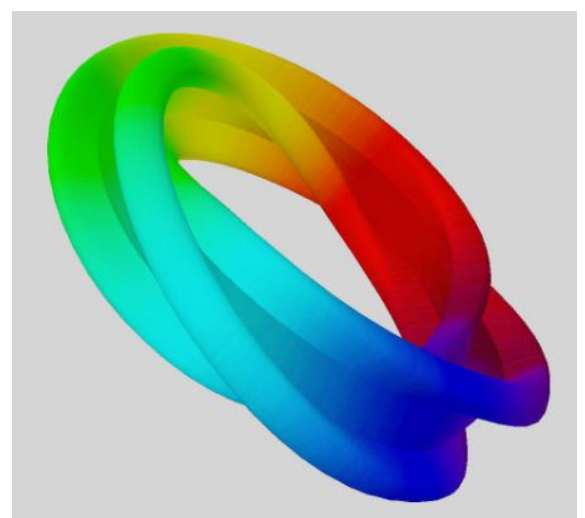

(a)

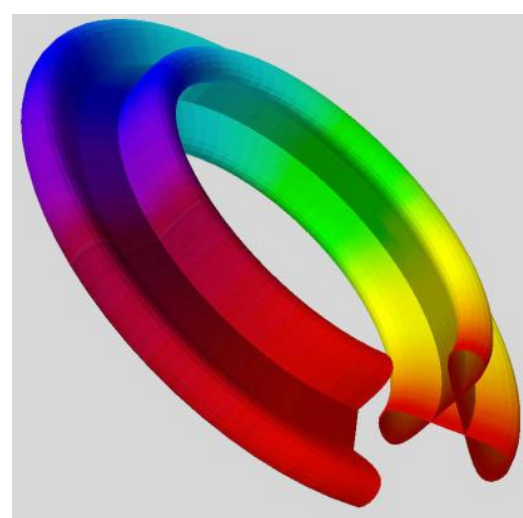

(b)

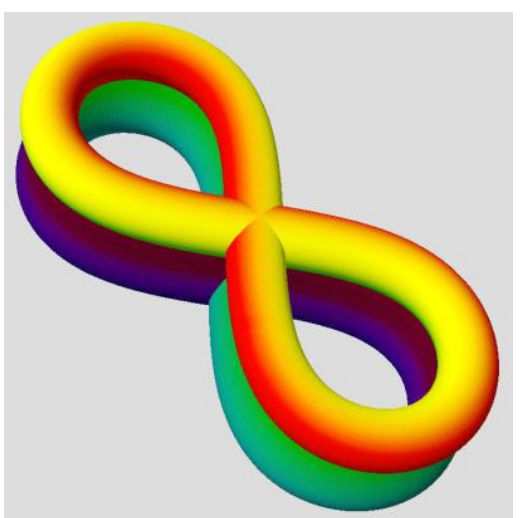

(c)

Figure 19. (a) Unwinding a doubly-rolled torus into a double loop with a figure-8 profile:

(a) starting state, (b) shown cut open and untwisted; (c) after parameter swap.
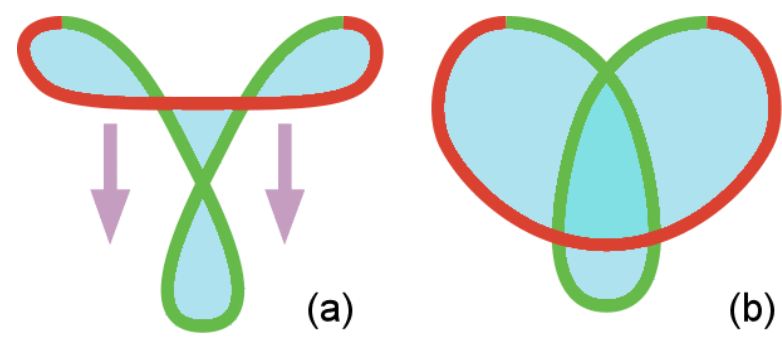

(b)



(c)

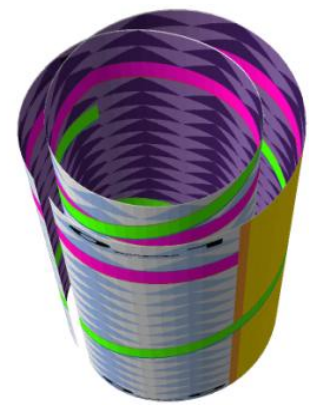

(d)

Figure 20. (a) $\rightarrow$ (c) Transforming a curtate hypocyclic profile into a double roll, (d) the resulting tube.

We use a transformation that combines a parameter swap with a profile change (Figures 13 and 14). But this time we start with $360^{\circ}$ of twist in the original structure, and, because of the doubly rolled tube, we need to show double walls everywhere (Figure 21). In the swap process, the double roll turns into a double loop. The $180^{\circ}$ flip of the crossed lobe (Figure $21(\mathrm{~b})$ ) removes the original twist of $360^{\circ}$, and the profile changes to a figure-8 shape (Figure 21(d)). This double loop can now be unfolded (Figure 18) without adding twist. Thus we end up with the generic representative of type T88 (Figure 19(c)).

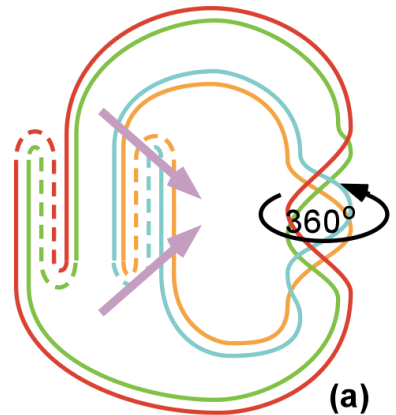



(b)

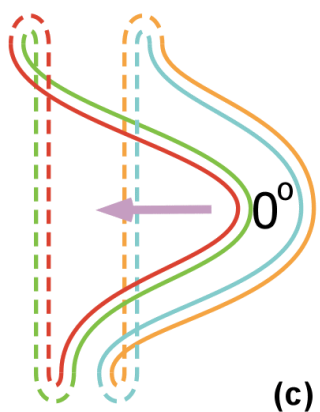

(c)

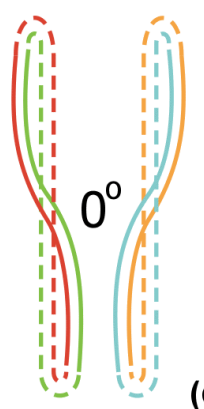

(d)

Figure 21. (a) $\rightarrow$ (d) Transforming a doubly-rolled torus (Figure 19(a)) into a torus with a double loop and with swapped parameterization (d), and eventually into a torus of type T88 (Figure 19(c)). 


\section{Tori with Collars}

Rather than containing a triple-fold as in Figure 11(a), the longitudinal torus profile could have a loop in it, which would then form a collar that exposes the inside of the surface (Figure 4(a)). Of course, tori can have more than one such collar, and collars could lie on the inside of the toroidal tube just as well as on the outside (Figure 4(b)). An operation based on the Dirac Belt Trick, nicely depicted in [7], allows us to remove such a collar and convert it into $360^{\circ}$ of M-twist in the torus tube. The principle is illustrated in Figure 22: as the belt is stretched, the loop in its middle turns into a full twist of the belt.


Figure 22. Left to right: Unwinding a loop in a ribbon into $360^{\circ}$ of twist using the Dirac Belt Trick [7].
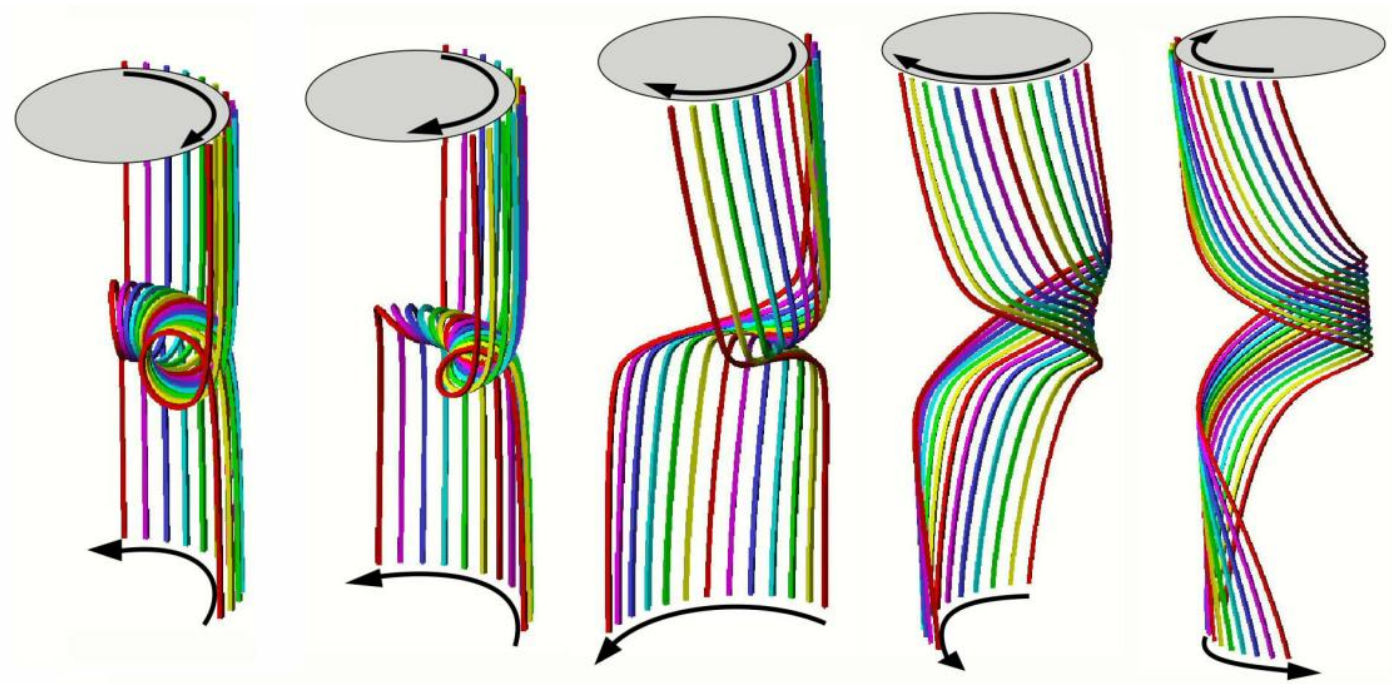

Figure 23. Left to right: Unwinding an internal collar into $360^{\circ}$ of M-twist. 
This fact can be used for the removal of a collar in the same way that it was used to evert a sphere in the movie Outside-In [10]. The surface near the collar is partitioned into sufficiently many corrugated, longitudinal rib-segments (eight were used in [10]), which are flexibly connected to one another through equally many "bellows." These bellows (not shown in Figure 23) provide the necessary flexibility, so that the surface can perform this un-winding move of the collar loop without experiencing any kinks. Figure 23 shows a collection of bands representing neighboring corrugations and illustrates conceptually how the collar can be un-done while jointly swinging one end of all the belts $360^{\circ}$ around the torus tube. At the end of this process, the ribbons form full $360^{\circ}$ helices, but lie nicely embedded in the cylinder surface. If there is more than one collar in a torus, each one can be turned into $\pm 360^{\circ}$ of twist; so pairs of collars can actually annihilate one another without creating any net twist.

\section{Tori with Internal Knots}

As a further artistic example of a torus, we look at the model in Figure 24. Here an ellipsoidal body is penetrated by an internal tunnel, which itself is knotted into a figure-8 knot (Figure 24(a)). The knot can be readily pulled apart into a straight tunnel, since torus branches can pass through one another. But the question arises, how much twist is produced in this process, and how much twist was there to begin with?
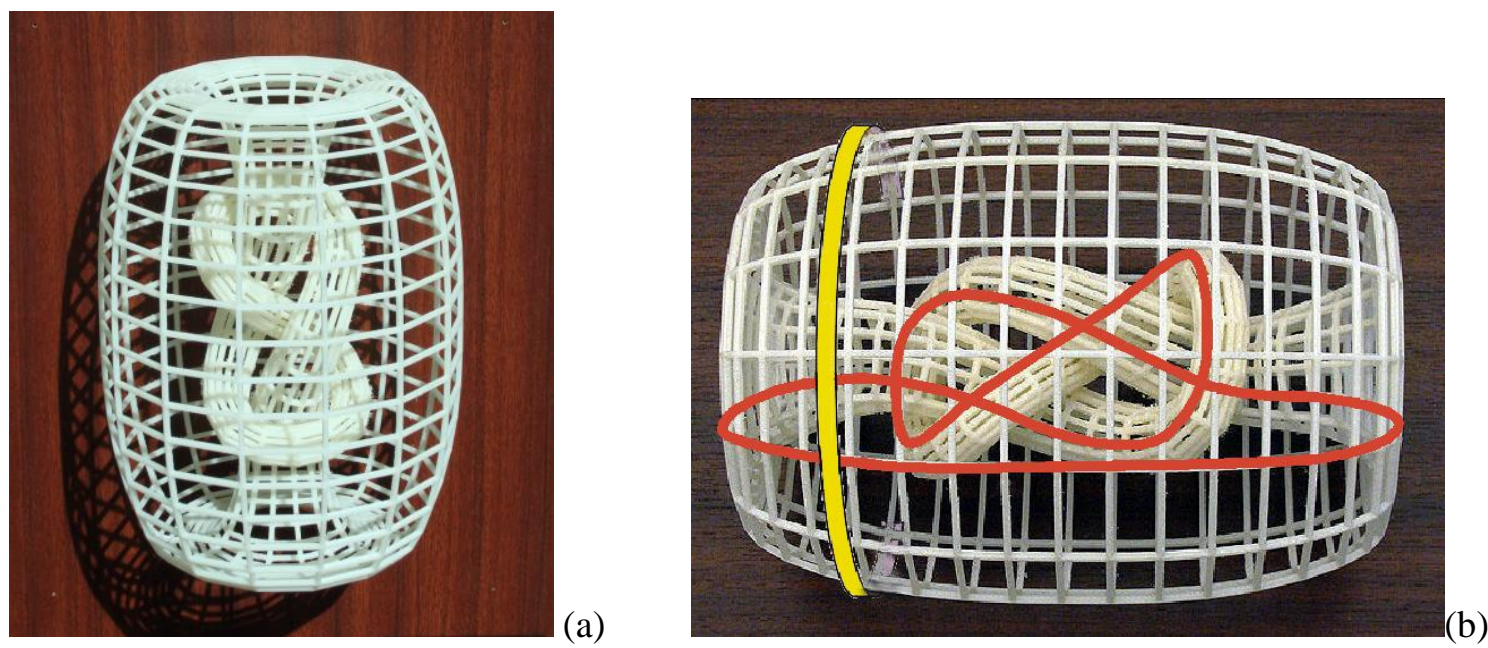

Figure 24: Torus with an internal figure-8 knot: (a) realized by fused-deposition modeling;

(b) analysis of the twistedness of the meridional (yellow) and longitudinal (red) parameter ribbons.

To answer these questions, the most direct approach simply analyzes the twistedness of the meridional and parallel grid lines. Looking at Figure 24(b), it is clear that the meridional bands are untwisted. The long, knotted gridlines that run parallel to the sweep path of this tube are more difficult to analyze. But it is possible to take a narrow paper-strip and bend it so that it follows the contortions of the surface along the path of one of these grid lines. Then one can tape the two ends of the strip together, stretch out the loop into a circle, and readily examine how much twist there is. In this example, there is no twist. Thus, since both complementary grid-line ribbons are without twist, this is just an ordinary torus of type TOO.

\section{Tori with Multiple Klein-Bottle Mouths}

Finally we look at a torus that at first glance might appear to be a Klein bottle on steroids (Figure 4(c)). Four mouth pieces of the classical Klein bottle are strung together in a cyclical manner. However, as long as there is an even number of them, so that there is an even number of inside-out surface reversals, this strange construction will topologically represent a torus. The analysis follows the reasoning outlined in the previous example of the internally knotted torus. Two of the parallels in Figure 4(c) have been colored white, so that one can follow them more easily and convince oneself that a corresponding surface ribbon again has no twist in it; thus this torus is also of type TOO. 


\section{6. "Optimal" Transformation Sequences: Parameter Swap}

From the above analysis we can conclude that swapping the parameters on an ordinary torus, i.e., exchanging the roles of parallels and meridians, keeps the torus in the same regular homotopy class TOO, since both characteristic ribbons remain untwisted in this process. Below I present an explicit transformation sequence that achieves this goal; but it is not as simple and elegant as I would like. Perhaps the difficulty of finding a direct, streamlined process is related to the fact that it is not obvious that one can indeed turn by $90^{\circ}$ the parameterization of an ordinary torus via a continuous, smooth, regular homotopy transformation, without cutting and re-gluing various ribbons.

\section{Looking for a Plausible Half-Way Point}

In my search for an appropriate transformation sequence I started by looking for a simple, symmetrical half-way point between the two desired end-states, i.e., a torus immersion that is equidistant (in a transformational sense) from two tori of type TOO, but with swapped parameterization. One plausible halfway point can be derived from two interlinked tori with complementary (swapped) parameterization. The two tori are made to touch in one point and are lined up in such a way that near the contact point their parametrizations, i.e., their (light) yellow and (dark) magenta ribbons coincide (Figure 25(a)). A saddle surface is constructed near the contact point, lying halfway between the two torus surfaces (Figure 25(b)). It can be seen as either two intertwined handles or as two tunnels touching each other. This type of Handle-Tunnel combination can also be constructed directly by taking the rectangular domain of the torus, bending it into a saddle surface, and joining the mid-portions of the horizontal and vertical edge pairs (Figure 25(b)).



(a)

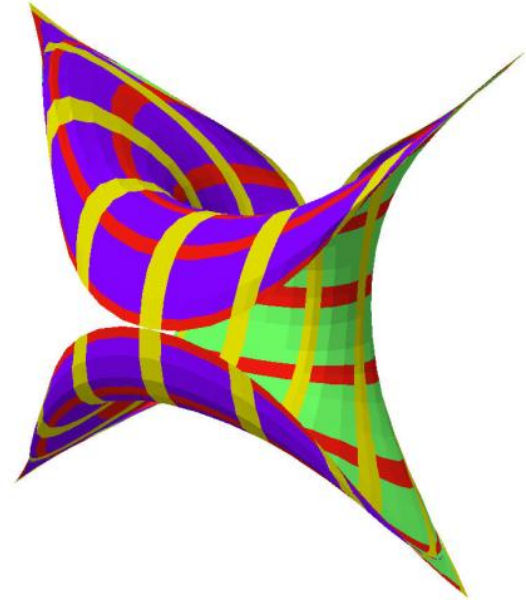

(b)

Figure 25. (a) Two interlinked tori. (b) Intermediate saddle surface formed near the contact point.

Now, if we choose to close the surface in Figure 25(b) by completing the torus colored light green on the outside, the magenta ribbons become meridians and the yellow ones turn into parallels. Alternatively, if we chose to complete the darker, purple torus, the red ribbons become the parallels, and the yellow ribbons turn into meridians - thus yielding a torus with swapped parameterization.

In Figure 26 this Handle-Tunnel element has been isolated and its boundary has been smoothed into a circle. The resulting surface patch is colored green on top (Figure 26(a)) and purple on its backside (Figure 26(b)). This entity is topologically identical to a torus with a hole in its surface (the location where the four corners of the rectangular domain are supposed to meet). To form a complete torus, this surface has to be closed with a membrane with the topology of a disk glued to its perimeter. Two ways of doing this are shown in Figure 26(c). One option is to use the purple hemispherical dome on top (shown as a transparent wire mesh); this leads to a torus with a purple tunnel. The other state of the torus is obtained if the lower, bowl-shaped closure is used (shown as a light green wire-mesh); this results in a 
torus with a green tunnel corresponding to an everted torus with swapped parameterization. Unfortunately, it is not possible to simply move this closing membrane from one extreme to the other without creating singularities with infinite curvature at the rim of the disk - because if we replace the Handle-Tunnel shape with a disk, we face the classical and difficult sphere-eversion problem.

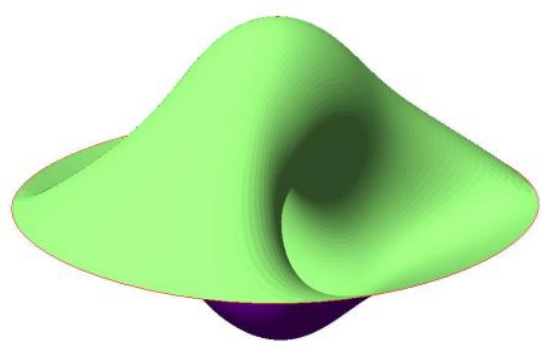

(a)

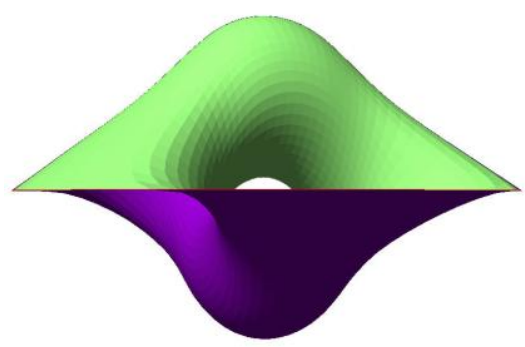

(b)

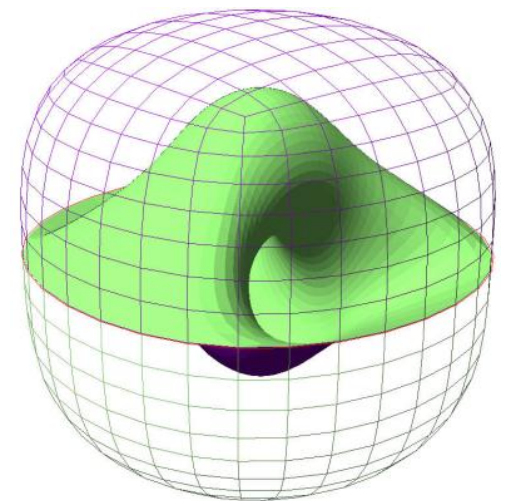

(c)

Figure 26. (a) Handle-Tunnel shape with circular perimeter; (b) side-view of Handle-Tunnel shape embedded in a disk; (c) conceptual model for parameter-swap with two alternative "closures".

\section{Using a Sphere-Eversion Process}

Because of this direct correspondence, we can use one of the published sphere eversion processes to move the closing membrane in Figure 26(c) from one position to the other. For a particularly nice visualization, I chose to stitch the disk containing the Handle-Tunnel geometry into a small circular hole at the "North pole" of an ordinary sphere (Figure 27(b)) and then apply the "Outside-In" eversion process [10] (Figure 27(a)). In this process the polar region is simply shifted to the other pole along the globe's axis. At the same time, the closing surface moves from lying below it to lying above it, thus accomplishing the desired parameter swap - unfortunately combined with an unwanted surface eversion. Thus, this transformation needs to be followed with a simple, straight torus eversion (Figure 11) to accomplish the desired net parameter swap in a torus of type TOO. Finding a more direct move sequence is one of the open challenges.
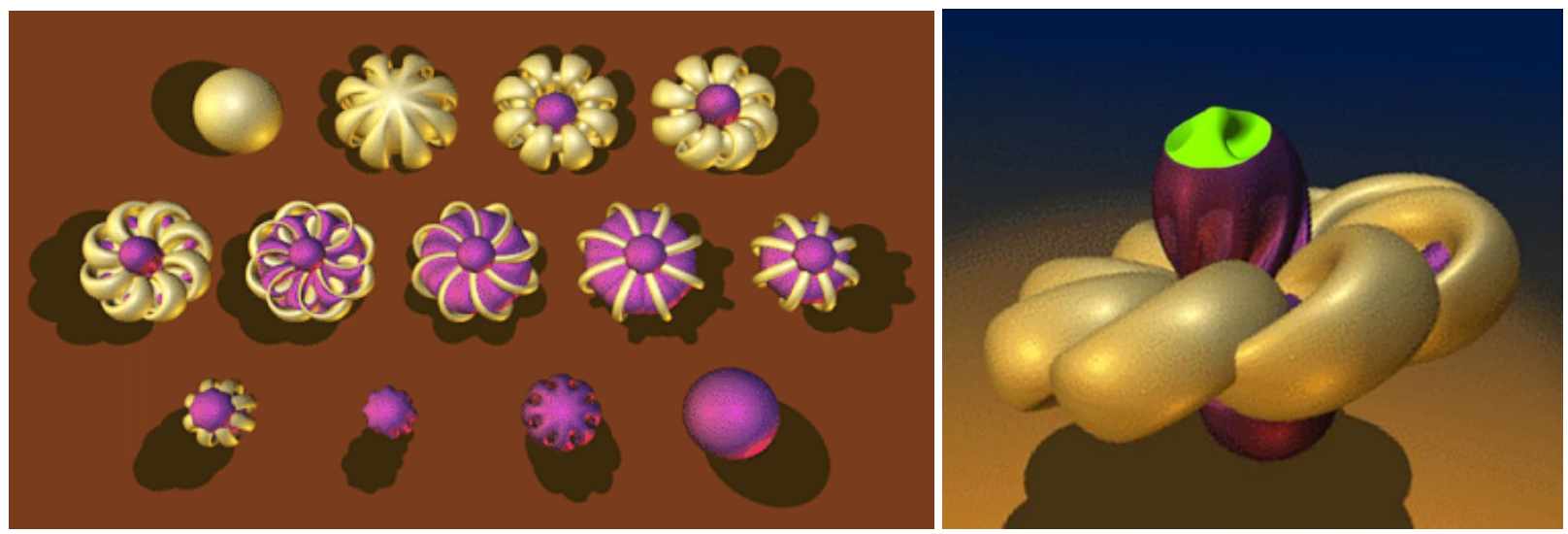

Figure 27. (a) Overview of the "Outside-In" sphere eversion process (from [11]; created by The Geometry Center; copyright 1995 University of Minnesota; used with permission). (b) The disk carrying the geometry of the two intertwined torus tunnels rides on top of this eversion process. 


\section{Visualization Models and Sculptural Prototypes}

When I construct a visualization model, I focus on clarity and aesthetics. Most importantly, the model must convey the mathematical insight that I want to illustrate. Most of the time, this can be done in many different ways. I aim to create a model that by itself, without any explanations of the mathematics behind it, will attract and intrigue the observer, and, in the best possible scenario, may even serve as a model for an aesthetically pleasing sculpture.

The process chosen to fabricate a particular model depends foremost on the requirements to yield a clearly understandable visualization. For the more complex torus immersions with many selfintersections, or for tori in which most of the relevant geometry occurs deep inside the overall shape, as in the case with the internal figure-8 knot (Figure 24), some way to make the torus surface transparent becomes crucial. Since, as of today, none of the layered manufacturing processes offer clear transparency, I often choose to represent the torus surface as a thin grid. This approach also allows me to show a representative set of parameter lines by means of the thin struts that form the grid. Ideally one would want to avoid any mutual intersections of grid struts where the torus surface may self-intersect, but for a complex model, where the surface is highly curved and intersects itself with a wide range of intersection angles, this can be very difficult to achieve with a strut width of a reasonable size needed for a robust visualization model. In a virtual visualization model the struts could be made very narrow, but of sufficient depth to make clear their relative ordering in space, so that the observer's brain can build a good mental 3D model. However, the tactile experience of a physical 3D model cannot yet be replaced with today's most advanced virtual reality technology.

Figure 28 presents possible half-way points for a torus eversion. These models are derived from Figures $11(\mathrm{c})$ and 12 by turning the Klein-bottle mouth on the right-hand side towards the outside of the loop, thereby introducing $180^{\circ}$ of twist into each of the two branches. For this model, color is essential to show that both sides of the torus surface point towards the outside world in equal amounts. The 3Dprinting process developed by Z-corporation, and now readily available for instance through Shapeways [21], is one of the few rapid prototyping techniques that can build such multi-colored parts (Figure 28(a)). Figure 29(b) shows the same basic structure, symmetrized and with minimal bending energy.

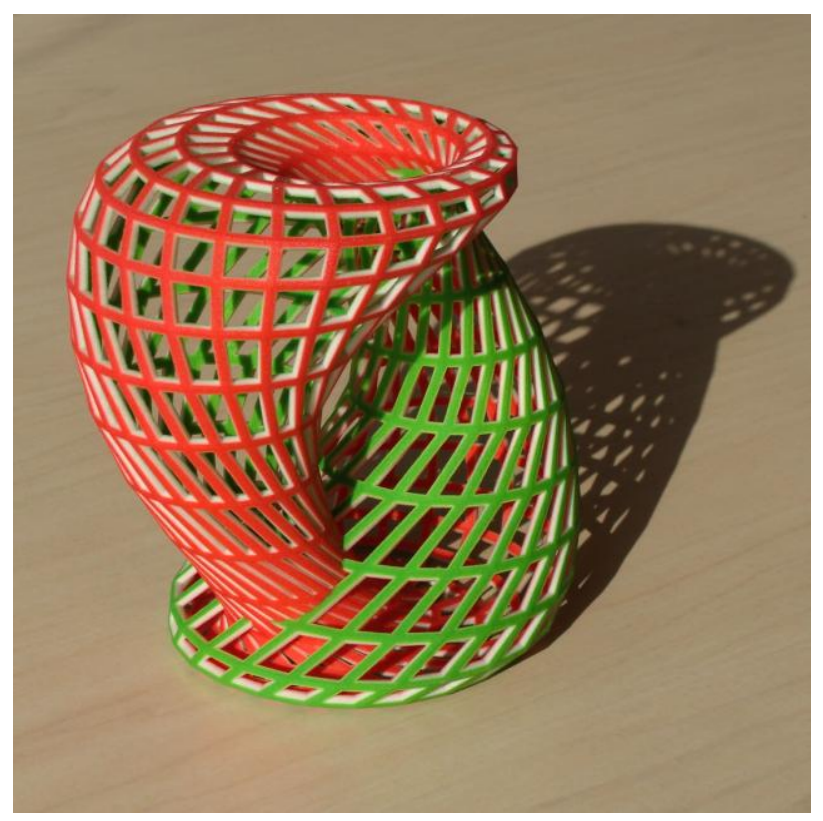

(a)

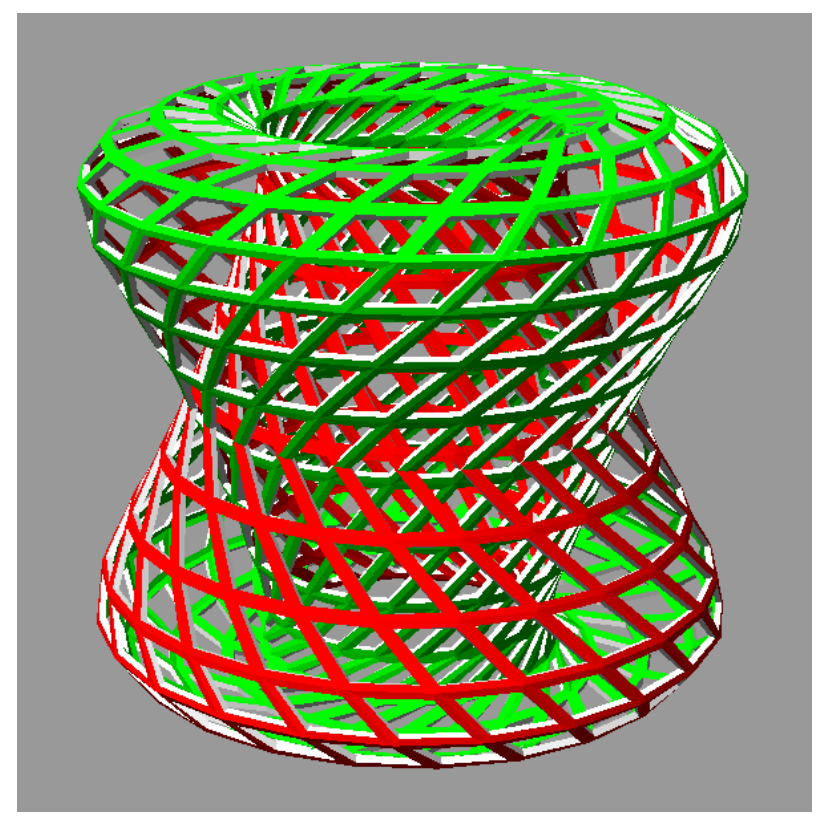

(b)

Figure 28: Symmetrical half-way points for a possible torus eversion process: (a) photo of a physical model derived from Figure 12, (b) computer rendering of a shape optimized for minimal bending energy. 
To conclude I present a torus model that was created with the primary goal of making an attractive sculpture - rather than attempting to elucidate any deep mathematical concept. TorusKnot_5-3 was introduced in summer 2010 at the Exhibit Art Inspired by Science at the Schneider Museum in Ashland Oregon, curated by Michael Crane [4]. The basic geometry of this sculpture is explained in Figure 29(a), where it is shown as a simple, regular rendering of a torus knot. It comprises a (cyan) strand, which itself has the topology of a torus, and which wraps around another conceptual (yellow) torus. The sculpture model of this knot is made interesting by giving the helical turns around the core torus a more triangular shape. Furthermore, the profile that generates the swept toroidal shape is not just a plain circle or a narrow rectangle, but rather a thin crescent shape, as can be found in many of Brent Collins' sculptures [3]. And finally, the scale of this cross section is varied by more than a factor of three as the path sweeps from inside the donut hole to the outside of the invisible torus.

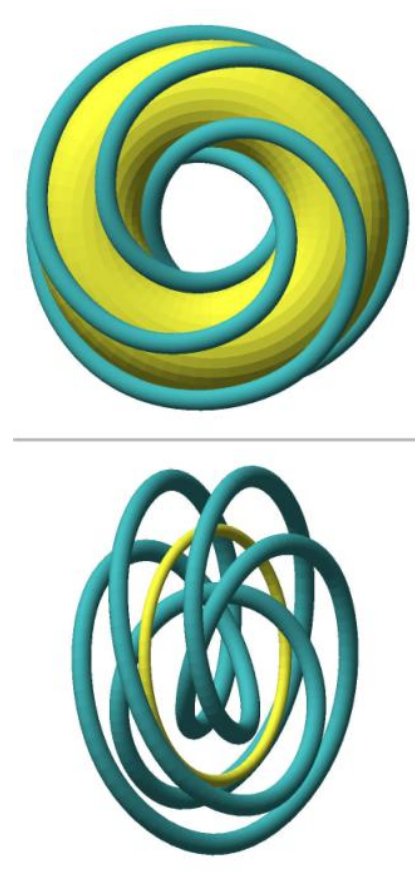

(a)

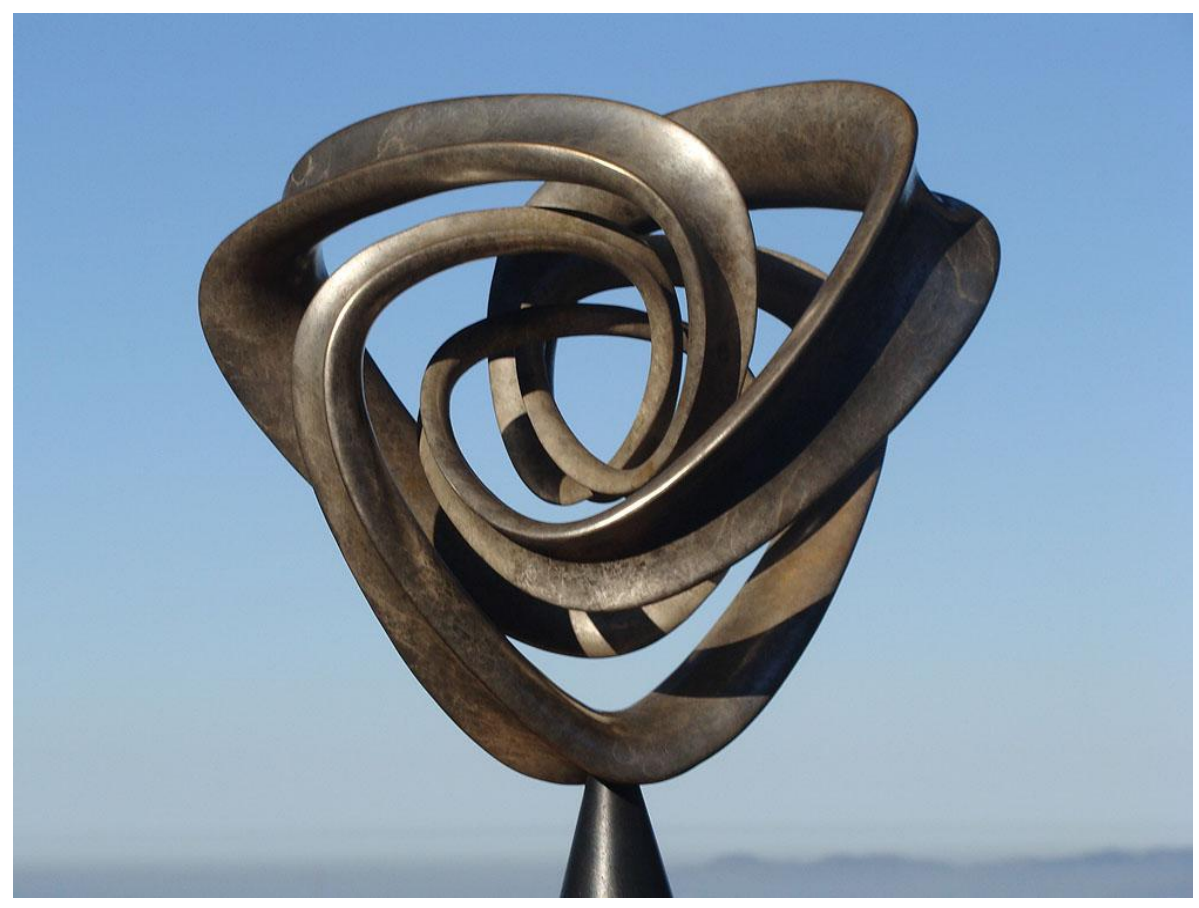

(b)

Figure 29. (a) Simple regular mathematical model of a $(5,3)$ torus knot: (top) tightly hugging the surface of a torus; (bottom) the torus knot strand seen from a different angle. (b) An artistic representation of the same knot designed by Carlo H. Séquin and cast in bronze by Steve Reinmuth [17].

The aesthetic considerations that I use to decide on the final form of a model are not easily captured in explicit rules. They are applied at two levels: first I need to determine the actual 3D shape and possible coloring of the model; then I need to choose a good angle and a suitable background to photograph the model for publication. Often I photograph a model against a natural blue sky, possibly with some light clouds (Figure 29(b)). But at other times, I lay the model on a contrasting background and photograph it in bright sun light, so that a distinct shadow is formed that conveys additional information about the shape of the 3D model (Figure 28(a)). For the 3D model itself I often choose as much symmetry as possible; but for the photograph to be published in a paper I rather choose an asymmetrical view, since such a view can often show many different aspects of the model in a single picture, relying on the observer's brain to use the perceived symmetry of the 3D shape for constructing the complete mental 3D model. 


\section{Summary and Conclusions}

To mathematicians tori manifest themselves in a much wider variety of forms than the classical doughnut shapes illustrated in Figure 1. Even allowing smooth geometrical transformations in which surfaces are allowed to pass through themselves, not all pairs of tori can be transfigured into one another. All possible immersions of marked (parameterized) torus surfaces in $\mathbf{R}^{\mathbf{3}}$ fall into four regular homotopy classes. Four simple, untwisted representatives for these four classes have been presented. They are easy to visualize and easy to remember. Several examples of regular homotopy-preserving transformations have been discussed. They should inform the reader how to analyze any arbitrary topological torus and identify the homotopy class that it belongs to; perhaps they can even help finding an explicit transformation sequence towards the corresponding representative.

However, work remains to be done to find the simplest, most elegant processes for some of the more important and intriguing transformations - in particular, the torus eversion and the parameter swap processes. Work has begun on the implementation of a special-purpose surface-optimization system that may allow us eventually to find some nice, stream-lined processes for these intriguing transformations.

One of the rewarding benefits of this investigation of complex toroidal structures is that one cannot avoid encountering many intriguing geometric shapes that need only a small amount of tweaking to be turned into attractive visualization models or even into large constructivist sculptures.

\section{Acknowledgements}

I would like to express my thanks to John Sullivan, Craig Kaplan, and to Matthias Goerner for insightful and constructive comments on preliminary versions of this paper. This work was supported in part by the National Science Foundation (NSF award \#CMMI-1029662 (EDI)).

\section{References}

[1] A. Chéritat, The torus inside out. - http://www.math.univ-toulouse.fr/ cheritat/lab/e_labo.html

[2] A. Chéritat, Torus eversion: turing a torus inside out. Video (49sec) http://www.youtube.com/watch?v=kQcy5DvpvlM

[3] B. Collins, S. Reinmuth, and C. H. Séquin, Design and Implementation of Pax Mundi II. ISAMA Proc. pp11-20, Texas A\&M, May 17-21, 2007.

[4] M. Crane, Art Inspired by Science, Schneider Museeum, Ashland, OR, summer 2010. http://www.cs.berkeley.edu/ sequin/ART/Science_Sculpture/

[5] Dehn Twist. From Wikipedia - http://en.wikipedia.org/wiki/Dehn_twist

[6] H. Ferguson, Sculpture. - http://www.helasculpt.com/

[7] G. K. Francis, A Topological Picturebook. Springer, New York. 1987. Chapter 7, Figs. 4 and 5.

[8] D. Harber, homepage. -- http://www.davidharber.com/sculpture/torus.htm

[9] J. Hass and J. Hughes, Immersions of Surfaces in 3-Manifolds. Topology, Vol 24, No.1, pp 97-112, 1985.

[10] S. Levy, D. Maxwell, D. Munzner, Outside-In. Video (22 min). http://www.geom.uiuc.edu/docs/outreach/oi/

[11] S. Levy, D. Maxwell, D. Munzner, Outside-In. Centerfold of Making Waves http://www.geom.uiuc.edu/docs/outreach/oi/centerfold.html

[12] macbuse, torus eversion $.-\mathrm{http}: / / \mathrm{www}$. youtube.com/watch? $\mathrm{v}=\mathrm{S} 4 \mathrm{ddRPvwcZI}$

[13] N. L. Max, Turning a Sphere Inside Out. International Film Bureau, Chicago, 1977. Video (21 min); reissued by AK Peters, 2004. - http://www.akpeters.com/sphere 
[14] B. Morin and J-P. Petit, Le retournement de la sphère. In Les Progrès des Mathématiques, pp 32-45. Editions Belin, Paris, France, 1980.

[15] A. Phillips, Turning a Surface Inside Out. Scientific American 214, pp 112-120, Jan. 1966.

[16] J. Prince, Jonathan Prince Studio. - http://www.jonathanprince.com/

[17] S. Reinmuth, Bronze Studio Inc. - http://www.reinmuth.com/

[18] J. Robinson, Symbolic Sculpture. - http://www.bradshawfoundation.com/jr/

[19] C. H. Séquin, Tori Story. Proc. Bridges, Coimbra, 2011, pp 121-130.

[20] C. H. Séquin, Torus Immersions and Transformations. Tech Report UCB/EECS-2011-83.

[21] Shapeways, Full color 3D printing. - http://www.shapeways.com/themes/full_color

[22] S. Smale, A Classification of Immersions of the Two-Sphere. Trans. Amer. Math. Soc. 90, pp 281290, 1959.

[23] J. M. Sullivan, G. Francis, and S. Levy, The Optiverse. In H-C. Hege and K. Polthier, eds, VideoMath Festival at ICM'98, Springer, 1998. Video (7 min).

[24] J. M. Sullivan, Conformal Tiling on a Torus. Proc. Bridges, Coimbra, 2011, pp 593-596.

[25] K. Ushio, homepage. - http://www2.memenet.or.jp/ keizo/ 